\title{
DUAL INHIBITORY ACTIVITIES OF ADHATODA VASICA AGAINST CYCLOOXYGENASE AND LIPOXYGENASE
}

\author{
Sagheer Ahmed ${ }^{1}$, Saima Gul ${ }^{2}$,Humera Gul ${ }^{3}$, Muhammad Hanif Bangash ${ }^{4}$ \\ 1. PAPRSB Institute of Health Sciences, University Brunei Darussalam \\ 2. Department of Zoology, Abdul Wali Khan University, Mardan, Pakistan \\ 3. Department of Botany, Abdul Wali Khan University, Mardan, Pakistan \\ 4. Chashma Nuclear Power Plant, Chashma city, Pakistan
}

Correspondence Author: sagheer.ahmed@ubd.edu.bn

\begin{abstract}
Adhatoda vasica (L.) Nees is a well-known plant drug in Ayurvedic and Unani medicine. It has been used for the treatment of various diseases, particularly for the treatment of inflammatory and cardiovascular diseases. However, the scientific rationale and mechanisms by which it functions in these diseases is not known. This study was designed to explore the inhibitory activity of Adhatoda vasica aqueous and butanolic fractions on arachidonic acid (AA) metabolism. For this purpose aqueous and butanolic fractions of Adhatoda vasica were screened for the presence of activities against arachidonic acid (AA) metabolites and their effectiveness was further evaluated by studying platelet aggregation induced by a AA, adenosine diphosphate (ADP), platelet activating factor (PAF), and collagen. AA metabolism was studied by thin layer chromatography system while platelet aggregation was measured by dual channel Lumi aggregometer. Aqueous fraction of Adhatoda vasica but not of butanolic fraction inhibited the AA metabolites through COX pathway $\left(\mathrm{TXB}_{2}\right)$ and LOX pathway (LP1 and 12-HETE). However, in platelet aggregation studies butanolic extract of Adhatoda vasica showed strong inhibition against AA, PAF and collagen induce aggregation but not against ADP. Aqueous fraction of Adhatoda vasica was active against none of the four aggregating agents. Adhatoda vasica possesses components which can inhibit AA metabolism and platelet aggregation. This may be one of the underlying mechanisms of their antiinflammatory effects.
\end{abstract}

\section{KEY WORDS}

Adhatoda vasica, bronchodilator, cyclooxygenase, lipoxygenase, aqueous fraction, butanolic fraction. Aggregation, aggregometer , inflammatory, bleeding piles, gingivitis, uterotonic, Radioactive, melanoma, isolation

\section{INTRODUCTION}

Adhatoda vasica (L.) Nees (family Acanthaceae) is a shrub 1-2.5 m high with opposite ascending branches. The leaves are simple, opposite, 7-19 cm long and 4-7 cm wide. It is also known under the common name Malabar nut tree and the Sanskrit name Vasaka. The flowers are white, pink or purple. The plant grows throughout the Indian peninsula up to an altitude of $1300 \mathrm{~m}$. The plant has been used in the traditional system of medicine in India for over 2000 years (Atal, 1980). It is a well-known drug in Ayurvedic and Unani medicine (Manjunath, 1948).

The frequent use of A. Vasica has resulted in its inclusion in the WHO manual The Use of Traditional Medicine in Primary Health Care, which is intended for health workers in South-East Asia to keep them informed of the therapeutic utility of their surrounding flora (World Health Organization, 1990). In this manual, A. Vasica is recommended for making sputum more fluid and for the treatment of cough, asthma and bleeding piles and it can be used for both adults and children for a long period. A methanolic extract from the entire plant has been shown to possess anti-allergic and anti-asthmatic activities in the guinea-pig after inhalation or intragastric administration at doses of $6 \mathrm{mg}$ per animal or $2.5 \mathrm{gm} / \mathrm{kg}$, respectively (Muller,1993). Ethanolic fraction from the leaves showed hypoglycaemic activity after oral administration in rats and rabbits (Modak, 1968), but had no effects on bacteria, fungi, yeasts or nematodes (Naovi,1991). The aqueous fraction, however, was shown to be active against the microbial flora isolated from patients with gingivitis (Patel, 1984). Vasicine showed bronchodilatory activity both in vitro and in vivo. Although vasicinone, the main metabolite of vasicine and also present in Adhatoda vasica extract, showed bronchoconstriction in vivo. The two alkaloids in combination showed a bronchodilatory activity both in vitro and in vivo (Atal, 1980). Vasicine was found to have uterotonic activity in different species including human beings. It was shown that the effect was influenced by the degree of priming of the uterus by estrogens. Vasicine initiated rhythmic contractions of human myometrial strips from both non-pregnant and pregnant uteri. The effect was comparable with that of oxytocin and methergin (Atal, 1980). However, there is no study that demonstrates the effects of Adhatoda vasica on arachidonic acid (AA) metabolism via cyclooxygenase (COX) and lipoxygenase (LOX) pathways. In the present investigation, studies are carried out on AA metabolism through COX and LOX pathways and effects on COX enzyme was further evaluated by studying human platelet aggregation.

\section{MATERIAL AND METHODS}

All organic solvents, chemicals and reagents used in the experiments were of analytical and highest purity grade. All the chemicals are purchased from Sigma chemical company, St. Louis, USA except 14C arachidonic acid which was purchased from Amersham Biosciences; acetic acid and citric acid from $\mathrm{BDH}$, sodium phosphate (mono and dibasic) from Merck and m-3M3FBS was purchased from Calbiochem (La Jolla, CA, USA).

\section{EXTRACTION OF PLANT MATERIAL}

Fresh rhizomes of Adhatoda vasica $(2.5 \mathrm{~kg})$ were collected in Swat District, N.W.F.P., Pakistan, and authenticated by Dr Humera Gul, Assistant Professor at the Department of Botany, University of Karachi, Pakistan. The rhizome of the Adhatoda vasica was ground using a commercial grinder to obtain fine powder. Five hundred (500) grams of ground material was soaked in 5 liter aqueous methanol and left for 24 hours at $4{ }^{\circ} \mathrm{C}$. The extract was filtered using a filtration flask and whattmann paper. This extract was evaporated on a rotary 
evaporator under reduced pressure $(-760 \mathrm{mmHg})$ to a thick, semisolid mass of dark brown color i.e. crude extract.

\section{FRACTIONATION OF THE CRUDE EXTRACT}

One hundred grams of the extract was dissolved in distilled water. This was then introduced in a separating funnel. $n$-hexane $(50-70 \mathrm{ml})$ was then added into the same separating funnel. This mixture was shaken vigorously, regularly allowing the air to escape out. It was kept for about $30 \mathrm{~min}$ to let the two layers separate. The upper layer of $n$-hexane was acquired and the same procedure was repeated twice and all the $n$-hexane layers were collected and concentrated in a rotary evaporator to obtain the $n$-hexane fraction. Butanol $(50 \mathrm{ml})$ was then added to the remaining layer and the same process was repeated as with $n$-hexane, finally obtaining the butanol fraction, the yield of both fractions was $12 \%$ and $42 \%$, respectively, while the remaining layer was filtered using a filtration flask and whattmann paper and used in the experiments as aqueous fraction.

\section{ARACHIDONIC ACID METABOLISM BY HUMAN PLATELETS}

Archidonic acid metabolism and thromboxane B2 (TXB2) formation was studied using a Berthold T.L.C. linear analyzer and chromatography data system (Model LKB 511, Berthold, W. Germany) as described previously (Saeed,2007). Human blood platelets were obtained in plastic bags containing 30-40 ml concentrated PRP from the diagnostic laboratory of the Aga Khan University, Karachi. The PRP was centrifuged at $1200 \mathrm{~g}$ for $20 \mathrm{~min}$ and the sedimented platelets were washed twice with an ice-cold phosphate buffer $(50 \mathrm{mM}, \mathrm{PH} 7.4)$, containing $\mathrm{NaCl}(0.15 \mathrm{M})$ and EDTA $(0.2 \mathrm{mM})$. After centrifugation platelets were re-suspended in the same buffer without EDTA at the initial PRP concentration. The PRP suspension was homogenized at $4{ }^{\circ} \mathrm{C}$ using a polytron homogenizer for $15 \mathrm{sec}$ and the homogenate centrifuged at $1200 \mathrm{~g}$ for $20 \mathrm{~min}$. Supernatant (300 $\mu 1$ containing $0.4 \mathrm{mg}$ protein) was incubated with $10 \mu \mathrm{g}$ unlabelled AA and $0.1 \mu \mathrm{Ci}[1-14 \mathrm{C}] \mathrm{AA}$ in the presence and absence of test fraction. After 15 min of gentle shaking in air at $37{ }^{\circ} \mathrm{C}$ the reaction was stopped by adding $0.4 \mathrm{ml}$ citric acid $(0.4 \mathrm{M})$ and ethyl acetate $(7.0 \mathrm{ml})$. After mixing and centrifuging at $600 \mathrm{~g}$ for $5 \mathrm{~min}$ at $4{ }^{\circ} \mathrm{C}$, the organic layer was separated and evaporated to dryness under nitrogen. Residues were dissolved in 40 $\mu 1$ of ethanol and $20 \mu \mathrm{l}$ was applied to silica gel $\mathrm{G}$ thin layer chromatography (TLC) plates (Analtech Delaware, USA). The AA, TXB2 (a stable degradation product of TXA2), LP1 and 12-HETE standards were plotted separately.

\section{SOLVENT SYSTEMS FOR DEVELOPING TLC PLATES}

The TLC plates were developed in ether/petroleum ether [boiling range 40-60]/acetic acid (50:50:1, v/v) to a distance of $17 \mathrm{~cm}$. By use of this solvent system the various lipoxygenasse products such as 12hydroxy-eicosatetraenoic acid (HETE) were separated with TXB2 and prostaglandins remaining at the origin. The solvent system used for the separation of TXB2 was ethyl acetate/iso-octane/water/acetic acid (11:5:10:2, v/v upper phase). Radioactive zones were located and quantified by use of a Berthold TLC linear analyzer and chromatography data system (Model LKB 511, Berthold, Germany).

\section{PREPARATION OF PLATELETS}

Platelet effects were studied in human platelets by taking blood via venipuncture from normal human volunteers reported to be free of medication for 7 days. Blood sample were mixed with $3.8 \%(\mathrm{w} / \mathrm{v})$ sodium citrate solution (9:1) and centrifuged at $260 \times \mathrm{g}$ for $15 \mathrm{~min}$ at $20{ }^{\circ} \mathrm{C}$ to obtain platelet-rich plasma (PRP). The remaining blood samples were centrifuged at $1200 \mathrm{x}$ g for 10 mins to obtain platelet poor plasma (PPP). Platelet count was determined by phase contrast microscopy and all aggregation studies were carried out at $37^{\circ} \mathrm{C}$ with
PRP having platelet counts between 2.5 and $3.0 \times 108 \mathrm{ml}-1$ of plasma.

\section{MEASUREMENT OF PLATELET AGGREGATION}

Aggregation was monitored using dual-channel Lumi- aggregometer (Model 400 Chronolog Corporation, Chicago, USA) using $0.45 \mathrm{ml}$ aliquots of PRP (Saeed 2007a,b). The final volume was made up to $0.5 \mathrm{ml}$ with the test fraction. Aggregation was induced by AA (1.7 $\mathrm{mM})$, ADP $(2.2 \mu \mathrm{M})$, PAF $(0.8 \mu \mathrm{M})$, collagen. The antiplatelet effects of test fractions were studied by pretreatment of PRP with each fraction for 2 min followed by addition of platelet agonist. The resulting aggregation was recorded for $5 \mathrm{~min}$ after challenge by the change in light transmission as a function of time. After establishing the anti-platelet activity against various agonists, dose-response curves were constructed to calculate the 1C50 values.

\section{RESULTS}

Aqueous fraction of Adhatoda vasica inhibited AA metabolism through COX as well as LOX pathways. However, butanolic fraction of Adhatoda vasica was completely inactive against COX product TXB2 as well as against LOX products LP1 and 12-HETE. Aqueous fraction of Adhatoda vasica showed IC50 values $(\mathrm{mg} \pm$ SEM) of $1.70 \pm 0.18$ against TXB2, $1.82 \pm 0.02$ against $\mathrm{LP} 1$ and

$1.42 \pm 0.10$ against 12 -HETE (see figure 1 and table 1 ).

Table 1: Table showing IC50 values of aqueous and butanolic fractions against selected AA metabolites and platelet agonists

\begin{tabular}{|c|c|c|c|c|}
\hline \multirow[t]{2}{*}{ Extracts } & \multicolumn{4}{|c|}{$\begin{array}{l}\text { IC50 values in mg against AA metabolites } \\
(\text { mean } \pm \text { SEM })\end{array}$} \\
\hline & TXB2 & \multicolumn{2}{|c|}{ LP1 } & 12-HETE \\
\hline Aqueous & $1.70 \pm 0.18$ & \multicolumn{2}{|c|}{$1.82 \pm 0.02$} & $1.42 \pm 0.10$ \\
\hline \multirow[t]{3}{*}{ Butanolic } & NA & \multicolumn{2}{|c|}{ NA } & NA \\
\hline & \multicolumn{4}{|c|}{$\begin{array}{l}\text { IC50 values in mg against platelet aggregation } \\
(\mathrm{mean} \pm \mathrm{SEM})\end{array}$} \\
\hline & $\overline{\mathbf{A A}}$ & ADP & PAF & Collagen \\
\hline Aqueous & NA & NA & NA & NA \\
\hline Butanolic & $0.32 \pm 0.14$ & NA & $0.28 \pm 0.064$ & $0.24 \pm 0.02$ \\
\hline
\end{tabular}

Figure 1: Inhibition of AA metabolism by the aqueous fraction of Adhatoda vasica, $(\mathrm{n}=4)$.

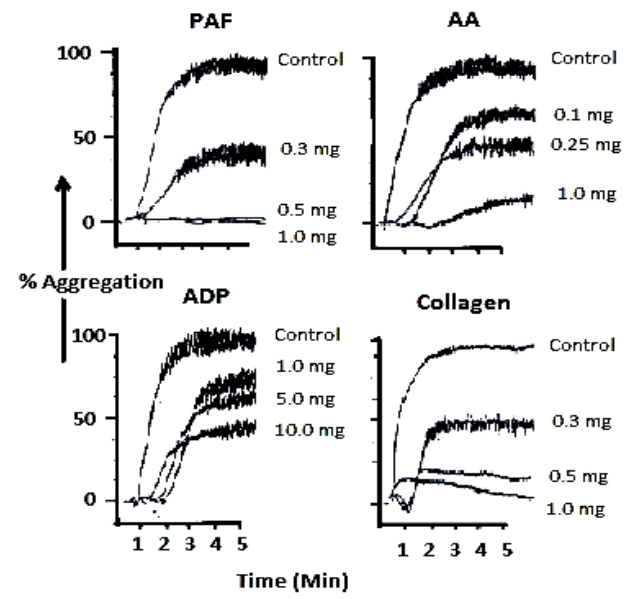

Figure 2: Butanolic fraction of the Adhatoda vasica inhibits platelet aggregation induced by various platelet agonists, $(n=4)$. 
Aqueous fraction of Adhatoda vasica up to $1.25 \mathrm{mg}$ showed very little effect on the inhibition of platelet aggregation induced by AA, ADP, collagen and PAF. However, butanolic fraction of Adhatoda vasica showed strong inhibition against AA, collagen and PAF while showing little effectiveness against ADP induced aggregation. Butanolic fraction of Adhatoda vasica inhibited platelet aggregation with IC50 value $(\mathrm{mg} \pm$ SEM) of $0.32 \pm 0.14$ against AA induced aggregation, $0.24 \pm 0.02$ against collagen induced aggregation and $0.28 \pm 0.064$ against PAF induced aggregation (see figure 2 and table 1 ).

\section{DISCUSSION}

The search for new compounds to control the AA metabolites is a promising area of research. Natural compounds produced by the secondary metabolism of plants are potentially important sources of dual inhibition of COX and LOX pathways of AA metabolism. The knowledge about natural inhibitors of AA pathway is scarce and practically non-existent for the control of various inflammatory conditions. In this study we screened aqueous and butanolic fractions of Adhatoda vasica for the presence of inhibitory constituents against AA metabolism and platelet aggregation.COX is the enzyme that catalyzes the rate-limiting step in prostaglandin synthesis, converting AA into prostaglandin $\mathrm{H} 2$, which is then further metabolized to prostaglandin E2, thromboxane A2 (TXA2) and other eicosanoids. When aqueous fraction of Adhatoda vasica was tested against COX pathway of AA metabolism, it was effective against COX metabolite TXB2. Butanolic fraction did not inhibit any of the metabolites. This indicates that COX inhibitory components are concentrated in the aqueous fraction of Adhatoda vasica. COX metabolites have been implicated in the pathophysiology of cardiovascular disease, cancer, and inflammatory diseases. Nonsteroidal anti-inflammatory drugs (NSAIDs) such as aspirin, acetaminophen, ibuprofen, indomethacin, and naproxen directly target COX. NSAID inhibition of COX is a useful tool in the treatment of inflammation and fever and, more recently, in the prevention of heart disease.

Lipoxygenases possess regiospecificity during interaction with substrates and on this basis have been designated as arachidonate 5-, 8, 12-, 15-lipoxygenase (5-LOX, 8-LOX, 12-LOX, and 15LOX)( Funk, 2001; Chandrasekharan,2002 \& Kuhn,1999). When aqueous fraction of Adhatoda vasica was tested against LOX pathway of AA metabolism, it was effective against 12-LOX metabolites LP1 and 12-HETE. Butanolic fraction inhibited neither LP1 nor 12-HETE. This indicated the presence of 12-LOX inhibitory components in the aqueous fraction of Adhatoda vasica. Platelet-type12-LOX have been found in different cancer tissues, including melanoma, prostate and epidermal cancers (Steele,1999). Evidence indicates that $12-\mathrm{LOX}$ is involved in both cancer cell proliferation and survival (Yamamoto,1999). Inhibition of 12-LOX with either 12-LOX inhibitors or a 12-LOX antisense oligonucleotide inhibits proliferation and induces apoptosis in carcinosarcoma cells, while adding back the 12-LOX metabolite, 12(S)-HETE prevents 12-LOX inhibitor-induced apoptosis (Tang,1997). Expression of 12-LOX is also correlated with tumor cell metastasis. 12(S)-HETE directly stimulates prostate cancer cell migration. Clinically, the degree of 12 -LOX expression in human prostate cancer correlates with the tumor grade and stage and 12 LOX expression level is higher in metastatic prostate cancers than in nonmetastatic ones (Nie, 2001).Platelets play a critical role in the initial restriction of blood loss following vascular injury by rapidly adhering to exposed subendothelial matrix components and aggregating to form a provisional plug. When aqueous fraction of Adhatoda vasica was tested against platelet aggregation induced by AA, ADP, PAF and collagen, it turned out to be ineffective. This was surprising because it was supposed to inhibit at least AA induced aggregation as it was able to inhibit TXB2 in the previous experiment. However, it is possible that aqueous fraction contains compounds which are stimulatory of platelet aggregation and hence the TXB2 inhibitory activity of aqueous fraction of
Adhatoda vasica is suppressed. When butanolic fraction of Adhatoda vasica was tested against platelet aggregation, it proved effective against AA, PAF and collagen induced aggregation whereas against $\mathrm{ADP}$ induced aggregation it was not active. It appears that AA metabolism inhibitory constituents are concentrated in the aqueous fraction of the Adhatoda vasica whereas platelet inhibitory constituents are concentrated in the butanolic fraction of the plant.

AA metabolism via COX and LOX pathways leads to the formation of a variety of metabolically active products with different roles in various inflammatory conditions. Our understanding of these roles is steadily increasing. This new information is providing a theoretical basis for development of new anti-inflammatory approaches targeted to COX and LOX activity. Since no effective inhibitor is currently available to block the COX and LOX pathways, aqueous fraction of Adhatoda vasica might be valuable for the isolation of pure compounds that show dual inhibition of COX and LOX pathways. This may prove to be extremely useful for treatment and prevention of a number of inflammatory conditions.

\section{REFERENCES}

- Atal, C. K. (1980). Chemistry and pharmacology of vasicine: A new oxytocic and abortifacient. Jammu: Regional Research Laboratory.

- $\quad$ Manjunath, B.L. (1948)The Wealth of India, A Dictionary of Indian Raw Materials and Industrial Products. Council of Scientific and Industrial Research, Delhi, pp. 31-32.

- World Health Organization, (1990) The use of traditional medicine in primary health care. A Manual for Health Workers in South-East Asia, SEARO Regional Health Papers, No 19, New Delhi, pp. 1-2.

- Muller, A. (1993). Chemistry and pharmacology of the antiasthmatic plants Galphimia glauca, Adhatoda vasica, Picrorhiza kurroa.

- Modak, A. K., \& Rao, M. R. (1966). Hypoglycaemic activity of a non-nitrogenous principle from the leaves of Adhatoda vasica Nees.

- Naqvi, S., Khan, M. S. Y., \& Vohora, S. B. (1991). Antibacterial, anti-fungal and anthelmintic investigations on Indian medicinal plants. Fitoterapia, 62, 221-228.

- $\quad$ Dhar, M. L., Dhar, M. M., Dhawan, B. N., Mehrotra, B. N., \& Ray, C. (1968). Screening of Indian plants for biological activity: Part I. Indian Journal of Experimental Biology, 6, 232-247.

- $\quad$ Patel, V. K., \& Venkatakrishna-Bhatt, H. (1984). In vitro study of antimicrobial activity of adhatoda vasika Linn.(leaf extract) on gingival inflammation--a preliminary report.

- Funk, C. D. (2001). Prostaglandins and leukotrienes: advances in eicosanoid biology. Science, 294(5548), 18711875 .

- Chandrasekharan, N. V., Dai, H., Roos, K. L. T., Evanson, N. K., Tomsik, J., Elton, T. S., \& Simmons, D. L. (2002). COX-3, a cyclooxygenase-1 variant inhibited by acetaminophen and other analgesic/antipyretic drugs: cloning, structure, and expression. Proceedings of the National Academy of Sciences,99(21), 13926-13931.

- $\quad$ Yamamoto, S., Suzuki, H., Nakamura, M., \& Ishimura, K. (1999). Arachidonate 12-lipoxygenase isozymes. In Lipoxygenases and their Metabolites (pp. 37-44). Springer US.

- $\quad$ Kuhn, H., Walther, M., \& Kuban, R. J. (2002). Mammalian arachidonate 15-lipoxygenases: Structure, function, and biological implications. Prostaglandins \& other lipid mediators, 68, 263-290.

- Steele, V. E., Holmes, C. A., Hawk, E. T., Kopelovich, L., Lubet, R. A., Crowell, J. A., ... \& Kelloff, G. J. (1999). Lipoxygenase inhibitors as potential cancer 
chemopreventives. Cancer Epidemiology Biomarkers \& Prevention, 8(5), 467-483.

- Tang, D. G., \& Honn, K. V. (1997). Apoptosis of W256 carcinosarcoma cells of the monocytoid origin induced by NDGA involves lipid peroxidation and depletion of GSH: Role of 12-lipoxygenase in regulating tumor cell survival.Journal of cellular physiology, 172(2), 155-170.

- $\quad$ Nie, D., Che, M., Grignon, D., Tang, K., \& Honn, K. V. (2001). Role of eicosanoids in prostate cancer progression. Cancer and Metastasis Reviews,20(3-4), 195206.

- Saeed, S. A., Ahmad, N., \& Ahmed, S. (2007). Dual inhibition of cyclooxygenase and lipoxygenase by human haptoglobin: its polymorphism and relation to hemoglobin binding. Biochemical and biophysical research communications, 353(4), 915-920.

- Saeed, S. A., Connor, J. D., Quadri, J., Tasneem, S., Ahmed, S., Mesaik, M. A., \& Choudhary, M. I. (2007). Inhibitors of phosphatidylinositide 3-kinase: effects on reactive oxygen specles and platelet aggregation. Pharmacological reports, 59(2), 238.

- $\quad$ Shah, B. H., \& Saeed, S. A. (1995). Phosphatidylinositol 3kinase inhibitor, wortmannin, inhibits 5-hydroxytryptaminemediated potentiation of platelet aggregation induced by epinephrine. Research communications in molecular pathology and pharmacology, 89(2), 157.

- $\quad$ Shah ,B.H., Shamim, G., Khan, S., \& Saeed, S.A. (1996) Protein kinase $\mathrm{C}$ inhibitor, chelerythrin, potentiates the adrenaline-mediated aggregation of human platelets through calcium influx. Biochem. Mol. Biol. Int. (38) 1135-41.

- $\quad$ Saeed, S.A., Simjee, R.U., Mehmood, F. (1993) Dual inhibition of platelet activation factor and Arachidonic acid metabolism by Ajmaline and the effect on carrageenaninduced rat paw edema, J. Pharm. Phrmacol. (45) 715-719.

- $\quad$ Saeed, S.A., Gilani, A.H., Simjee, R.U., Shah, B.H. (1997) Antithrombotic and anti inflammatory effects of protopin, Pharmacol. Res. 36 1-8. 